\title{
Análisis financiero en el sector industrial con aplicación de herramientas de la lógica borrosa @() (1) (1)
}

\section{Financial analysis in the industrial sector with application of fuzzy logic tools}

Kléber Antonio Luna Altamirano', Juan Ennis Espinoza González², William Henry Sarmiento Espinoza ${ }^{3}$, Celio Froilán Andrade Cordero ${ }^{4}$, Verónica Paola Chamba Esparza ${ }^{5}$.

\begin{abstract}
DOI: https://doi.org/10.33262/cienciadigital.v3i2.3.566

The financial analysis gains importance in the accounting and administrative field, this one contributes with information on the financial situation of the company, exposing the correct thing or not of the management, the problem of the companies of the industrial sector of the city of Cuenca-Ecuador, is the ignorance of new forms of financial analysis with the purpose of knowing its real financial situation. The objective is to provide managers with a new way of calculating the most important financial ratios, with the support of fuzzy logic, applying confidence intervals by means of a range of values, with the purpose of limiting the uncertainty within this analysis, in order to reduce the subjectivity or risk that traditional, ambiguous and imprecise calculation may have. The results of the application of this avant-garde tool describe the variability between the value obtained ratio and the real value thereof. Thus, in a confidence interval it indicates that, within the established range, the real value of the financial ratio is found, increasing the objectivity of the result.
\end{abstract}

Keywords: Fuzzy logic, financial ratios, industry sector.

\footnotetext{
1 Universidad Católica de Cuenca, Unidad Académica de Administración, Cuenca, Ecuador. klunaa@ucacue.edu.ec

2 Universidad Católica de Cuenca, Maestrante en Administración de Empresas, Cuenca, Ecuador. jespinozag@psg.ucacue.edu.ec

${ }^{3}$ Universidad Católica de Cuenca, Unidad Académica de Administración, Cuenca, Ecuador. wsarmiento@ucacue.edu.ec

${ }^{4}$ Universidad Católica de Cuenca, Unidad Académica de Administración, Cuenca, Ecuador. candrade@ucacue.edu.ec

${ }^{5}$ Universidad Católica de Cuenca, Maestrante en Contabilidad y Auditoría con Mención Tributaria, Cuenca, Ecuador.vpchambae279@psg.ucacue.edu.ec
} 


\section{Resumen}

El análisis financiero cobra importancia en el campo contable y administrativo, este aporta con información sobre la situación financiera de la empresa, exponiendo lo correcto o no de la gestión, el problema de las empresas del sector industrial de la ciudad de Cuenca-Ecuador, es el desconocimiento de nuevas formas de análisis financiero con el propósito de conocer su situación financiera real. El objetivo es entregar a los directivos una nueva forma de cálculo de los ratios financieros más importantes, con el apoyo de la lógica borrosa, aplicando intervalos de confianza mediante rango de valores, con el propósito de acotar la incertidumbre dentro de este análisis, con ello se trata de reducir la subjetividad o riesgo que puede tener el cálculo tradicional, ambiguo e impreciso. Los resultados de la aplicación de esta herramienta de vanguardia describen la variabilidad entre el valor obtenido ratio y el valor real del mismo, así, en un intervalo de confianza indica que, dentro del rango establecido, se halla el valor real del ratio financiero, incrementando la objetividad del resultado.

Palabras clave: Lógica borrosa, ratios financieros, sector industrial.

\section{Introducción}

El análisis financiero por medio de ratios es un instrumento valioso para la toma de decisiones dentro de las organizaciones. Para los directivos y la alta gerencia de una empresa es importante conocer la situación financiera en la que se encuentra, para ello se hace necesario analizar los estados financieros por medio de ratios.

El problema de la presente investigación es el desconocimiento de nuevas formas de análisis de la situación financiera por parte de las empresas del sector industrial de la ciudad de Cuenca-Ecuador, cuyo objetivo es entregar a estas organizaciones una nueva manera de analizar la situación financiera de sus empresas, a través de ratios con enfoque en la lógica difusa, con la aplicación de herramientas de vanguardia como son los intervalos de confianza (bandas), en donde se trata de reducir la incertidumbre determinando el valor de mayor probabilidad de ocurrencia del fenómeno. Casanovas y Fernández (2003) afirman: "Podemos definir a un intervalo de confianza como un dato incierto que sirve para predecir el valor de cierta variable entre dos extremos, uno inferior y uno superior" (p.20).

Los mismos autores Casanovas y Fernández (2003) explican que un subconjunto borroso es el resultado de asignar a los valores de cierta variable de presunción, posibilidad o probabilidad. Dentro de la metodología se analiza los ratios financieros más importantes partiendo del trazo triangular, cuyo análisis será desde la perspectiva de un sub conjunto borroso triangular, en donde se admitirá la linealidad de los niveles de presunción o alfa cortes entre los valores que lo precisen. Partiendo de este análisis, se puede proyectar estas razones financieras a futuro, dejando a los directivos hacer el esfuerzo necesario para que 
este valor este dentro de intervalo de confianza proyectado, logrando de esta manera romper el cálculo tradicional para una mejor gestión empresarial.

\section{Estado del arte}

El análisis financiero parte del análisis de los estados financieros a través de ratios o razones financieras, este evalúa la situación financiera actual y anterior de una organización, determinando estimaciones y predicciones sobre entornos a futuro. Sobre ello algunos autores han publicado sus investigaciones, entre ellos se tiene: Bernal y Amat (2012) describen los ratios financieros ideales con mayor potencial predictivo y sus fórmulas, así como el diseño de un instrumento de investigación para medir la pertinencia de la publicación. Arimany, Farreras y Rabaseda (2016) presentan los principales indicadores económicos y financieros para poder diagnosticar la salud de estas empresas en el periodo 2008-2013 mediante un análisis a corto y largo plazo, un análisis de los resultados y un análisis de los cambios patrimoniales y de los flujos de efectivo de las empresas vinícolas, también realizan una comparativa con las empresas vinícolas catalanas para el mimo periodo.

Villegas, Hernández y Salazar (2017) explican el coeficiente de valor añadido intelectual, junto con sus componentes eficiencia del capital empleado, eficiencia del capital humano y eficiencia del capital estructural, se emplean como una herramienta que permite analizar su correlación y su influencia en factores como la rentabilidad, la capitalización de mercado y el valor de las acciones, en un grupo de empresas mexicanas del sector industrial que cotizan en la Bolsa Mexicana de Valores, lo que permitirá identificarlos como elementos claves que contribuyen en su competitividad y éxito empresarial. Nava (2009) analiza la importancia del análisis financiero como herramienta clave para una gestión financiera eficiente, explica que este se basa en el cálculo de indicadores financieros que expresan la liquidez, solvencia, eficiencia operativa, endeudamiento, rendimiento y rentabilidad de una empresa, considera que una empresa con liquidez es solvente pero no siempre una empresa solvente posee liquidez, indica que el análisis financiero basado en cifras ajustadas por inflación proporciona información financiera válida, actual, veraz y precisa.

La lógica difusa, conocida también con el nombre de lógica borrosa, dio sus inicios en el año 1965 de la mano de Lotfi Asker Zadeh con su publicación "Fuzzy Sets", en donde se adoptan los conceptos de la lógica y de los subconjuntos borrosos, a través de la definición de grados de pertenencia. Otros autores como Kaufmann y Gil (1986). Introducción de la teoría de subconjuntos borrosos a la gestión de las empresas. Rico y Tinto (2008) explica sobre la matemática borrosa y su utilización en varios modelos, para diferentes casos de aplicación en las ciencias económicas y administrativas, permiten señalar la importancia que cobra, para efectos de mejorar el tratamiento y la calidad de la información contable y financiera al igual que los procesos de toma de decisiones, la incorporación de variables como la subjetividad, 
la borrosidad y la imprecisión, en la construcción, diseño y estudio de dinámicas asociables a los sistemas de información.

Medina (2006) recopila el estado actual de las aplicaciones de la teoría de conjuntos difusos y los sistemas de inferencia difusos en la solución de problemas financieros, además, se realiza una crítica de los modelos tradicionales de toma de decisiones financieras, que no captan de forma clara las dinámicas del comportamiento de los mercados. Díaz et al. (2017) evalúan los ratios del segmento 1 de las Cooperativas de Ahorro y Crédito del Ecuador en un rango de bajo, estable y óptimo, mediante grados de pertenencia y variables lingüísticas que sustentan la conversión de los índices financieros a términos difusos, toman en consideración los principales ratios del sector cooperativo, basados en las metas del sistema CAMEL, los cuales a través de la función triangular de la lógica difusa obtienen grados de pertenencia donde se sitúan los ratios del segmento. Luna et al. (2018) demuestran la rentabilidad para comercializar en el mercado Gualacense un modelo novedoso de calzado para dama con la utilización de la lógica difusa, aplicando herramientas de avanzada como alfa cortes en los datos obtenidos de los expertos artesanos del calzado; y, la aplicación de números borrosos triangulares (NBT) en forma de intervalos (bandas) con el propósito de trabajar a ciertos niveles de posibilidad de que ocurra el fenómeno.

Finalmente, Kaufmann y Gil-Aluja (1987) en su obra Técnicas operativas de gestión para el tratamiento de la incertidumbre, dan a conocer que un número borroso es considerado como una secuencia finita o infinita de intervalos de confianza, obra trascendente para el acrecentamiento del conocimiento dentro de la lógica difusa (fuzzy logic).

\section{Metodología}

El análisis financiero dentro de toda organización, es considerada como el conjunto de procedimientos usados para diagnosticar la situación financiera, con el propósito de tomar decisiones correctas en el ámbito empresarial. Para Villegas (2002), el análisis financiero consiste en evaluar la posición financiera y los resultados de una empresa con relación a sus operaciones, cuya finalidad es descubrir las fortalezas y debilidades de la misma.

En la presente investigación se desarrolla herramientas de avanzada que ofrece la lógica difusa, aplicadas a los ratios financieros más importantes dentro del sector industrial de la ciudad de Cuenca Ecuador. Para ello se aplica dentro de la metodología números borrosos triangulares e intervalos de confianza (bandas), Gutiérrez (2006) explica que un número borroso asocia dos conceptos: el de intervalo de confianza, que se encuentra ligado a la noción de incertidumbre y el de nivel de presunción ligado a la percepción del individuo, es decir, a la noción de valuación. Kaufmann y Gil-Aluja (1987) dan a conocer que un número borroso triangular (NBT) que es aquel subconjunto borroso que se forma por una secuencia 
finita e infinita de intervalos de confianza, que surgen de asignar un nivel de confianza a los valores de un conjunto referencial dado el que define su grado de pertenencia. Los mismos autores, Kaufmann y Gil Aluja (1986) manifiestan que el uso de números borrosos triangulares en el tratamiento de la incertidumbre dentro de la organización es conocido desde los inicios de la incorporación de la lógica fuzzy en los problemas empresariales.

Los ratios financieros que se analizan en este estudio, son los más importantes dentro del sector industrial, entre ellos se pueden enunciara a la liquidez, prueba acida y solvencia, cada uno de ellos se los analizara con enfoque en la lógica borrosa, con la finalidad de reducir la incertidumbre, vaguedad e imprecisión en la información.

\section{Ratio de Liquidez: Razón corriente}

Esta razón financiera explica la capacidad que tiene la organización para cumplir con sus deudas, obligaciones financieras, o pasivos a corto plazo, para determinar se divide el activo corriente entre el pasivo corriente, indica cuantas deudas de corto plazo son cubiertas por los activos respaldados en efectivo en el mismo plazo, lo ideal es que su respuesta sea mayor a uno, si el resultado es muy superior a la unidad, este se convierte en activos corrientes no productivos. La tabla 1 indica un historial por años de los resultados de este ratio financiero.

Tabla 1. Razón de Liquidez en años

\begin{tabular}{ll}
\hline Historial Años & Valor \\
\hline & 2,83 \\
2014 & 2,15 \\
2015 & 3,47 \\
2016 & 2,52 \\
2017 & 1,97 \\
2018 & \\
\hline
\end{tabular}

Fuente: Elaboración propia

Con la información presentada en la tabla 1, se procede a determinar los niveles mediante la aplicación geométrica de un triángulo, en el cual se calcula los niveles pesimista, ideal y optimista a partir de la lógica borrosa, con ello se logra determinar intervalos de confianza (bandas), a partir de alfa cotes. Casanovas y Fernández (2003) afirman: "Podemos definir un intervalo de confianza como un dato incierto que sirve para predecir el valor de cierta variable, uno inferior y otro superior" (p.20). Lo enunciado se detalla en el siguiente gráfico. 
Gráfico 1. Análisis del ratio liquidez: razón corriente bajo el enfoque difuso

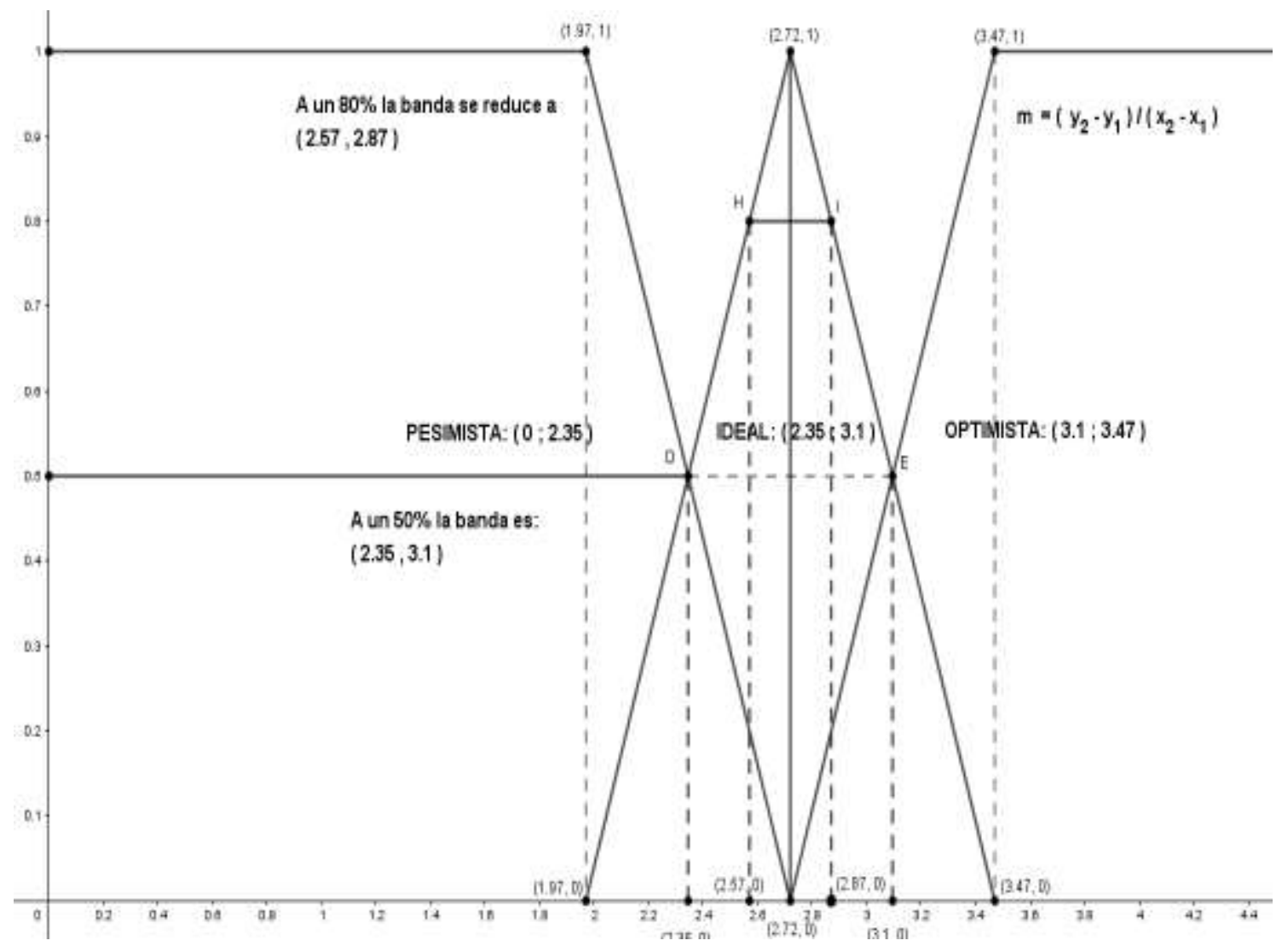

Fuente: Elaboración propia

Con este trazo geométrico, se determina en primer lugar un numero borroso triangular (NBT), con el propósito de determinar los tres niveles explicados anteriormente. Los mismos autores Casanovas y Fernández (2003) afirman: "la tripleta es también un dato incierto. Sin embargo, viene definida por dos extremos y un valor central. Mientras los extremos inferior y superior acotan el valor previsional de la variable en cuestión, el valor central nos indica el valor que tiene mayor posibilidad de cumplirse" (p.20-21).

En el siguiente gráfico, se demuestra el numero borroso triangular (NBT), conformado por [1.97, 2,72, 4.47], el valor del extremo izquierdo corresponde a la razón corriente del año 2018, el valor del extremo derecho al año 2016 referente al ratio de liquidez, y el valor central representa la máxima posibilidad de ocurrencia de este ratio. Lo explicado se demuestra en el gráfico 2. 
Gráfico 2. Intervalos de confianza con alfa cortes $(\alpha)$

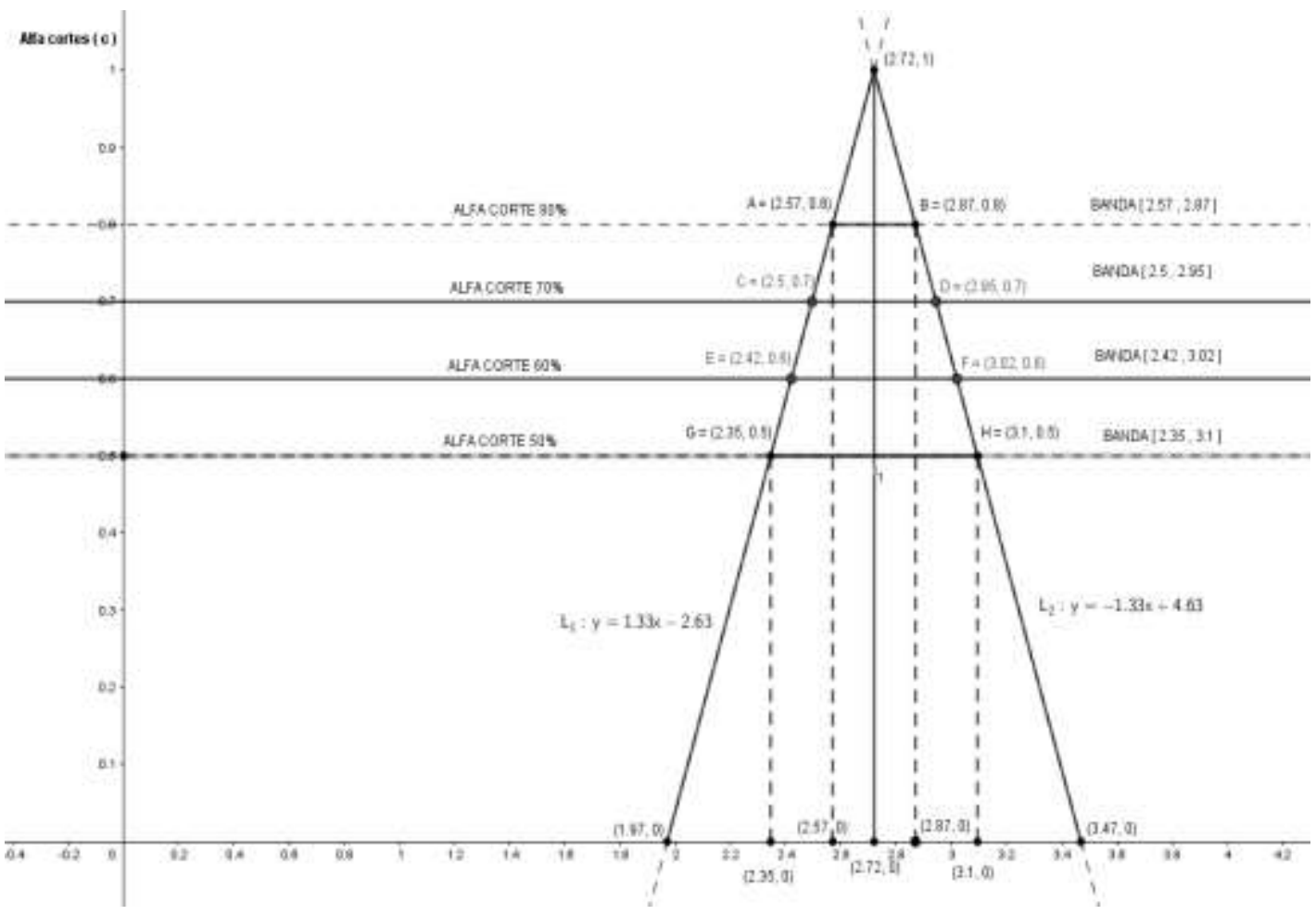

Fuente: Elaboración propia

Apegándose al análisis matemático, la ecuación de la recta $l_{1}$ se obtiene a través de la fórmula:

$y-y_{1}=m\left(x-x_{1}\right)$ donde, $m=\frac{y_{2}-y_{1}}{x_{2}-x_{1}}$; siendo:

$\left(x_{1}, y_{1}\right)=(2.72,1) y\left(x_{2}, y_{2}\right)=(1.97,0)$, sustituyendo los valores la ecuación de la recta que pasa por los dos puntos se obtiene:

$y=1.33 x-2.63$

Si alfa corte corresponde al $80 \%$ y este valor se sustituye en y, la abscisa resultante es:

$0.8=1.33 x-2.63$

$x=2.57$, este valor corresponde a la banda izquierda. 
De forma análoga se obtiene la ecuación de la recta $l_{2}$

$y=-1.33 x+4.63$

$0.8=-1.33 x+4.63$

$x=2.87$, valor correspondiente a la banda derecha.

Este nuevo análisis con lógica difusa, del ratio financiero razón corriente de liquidez, da la posibilidad de entender, que mientras el alfa corte $(\alpha)$ se aproxima al $100 \%$ el intervalo de confianza se reduce. Considerando un alfa corte del $50 \%$ [2.35, 3.10] y otro del $80 \%$ [2.57, 2.87], se reduce la incertidumbre y posibilita una serie de decisiones importantes para la alta gerencia en función de los niveles óptimos definidos mediante datos históricos de la empresa.

En forma análoga, se presenta el análisis de los ratios financieros prueba ácida y solvencia, estos representan los más importantes conjuntamente con el anterior, para las empresas del sector industrial de la ciudad de Cuenca-Ecuador.

\section{Ratio de Liquidez: Prueba ácida}

Este indicador financiero, determina con mayor exactitud la capacidad que tiene la empresa de cancelar sus obligaciones corrientes, por intermedio de sus activos corrientes, sin contar con sus existencias o inventarios, únicamente con recursos en efectivo, cartera e inversiones temporales. Lo ideal de este ratio financiero es que su resultado sea superior a la unidad, en el caso de ser un alto, la empresa contaría con exceso de recurso corriente y estaría afectando su rentabilidad. La tabla 2 y el grafico 3, explican lo enunciado.

Tabla 2. Prueba Ácida en años

\begin{tabular}{ll}
\hline Historial Años & Valor \\
\hline & 1,38 \\
2014 & 1,69 \\
2015 & 1,74 \\
2016 & 1,42 \\
2017 & 1,26 \\
2018 & \\
\hline
\end{tabular}

Fuente: Elaboración propia 
Gráfico 3. Análisis del ratio liquidez: prueba acida bajo el enfoque difuso

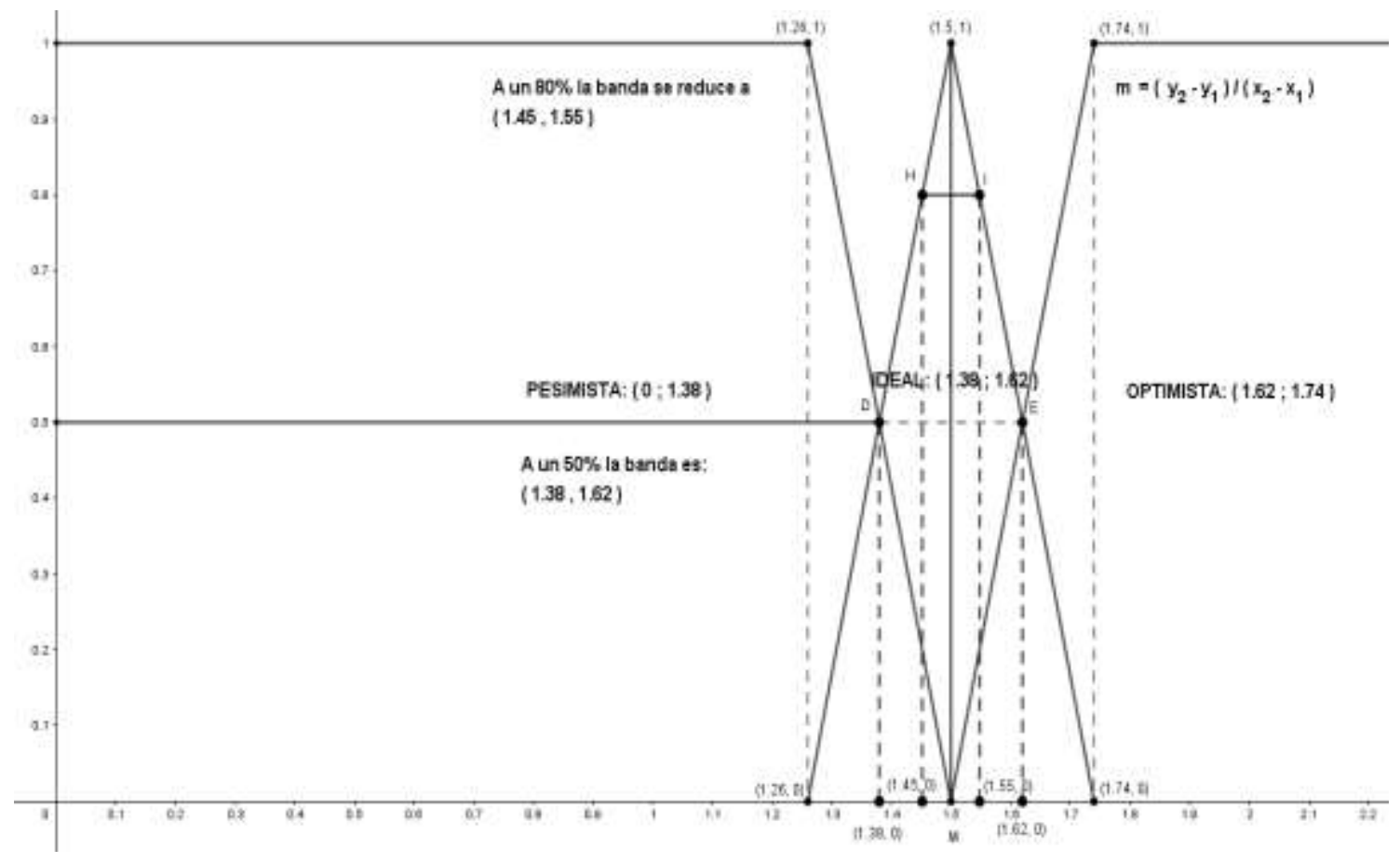

Fuente: Elaboración propia

Se determina el numero borroso triangular (NBT), a partir de la tripleta [1.26, 1,50, 1.74], donde: el valor del extremo izquierdo concierne al historial del año 2018, el valor del extremo derecho, corresponde al año 2016 referente a la razón financiera de la prueba acida, y el valor del centro constituye la máxima posibilidad de ocurrencia de este indicador financiero. A partir del trazo triangular se realiza el mismo análisis anterior con relación a los intervalos de confianza.

\section{Ratio de solvencia}

Este indicador financiero, se expresa mediante la capacidad de pago que tiene la empresa frente a todos sus compromisos o deudas a largo plazo. Una organización se considera solvente cuando sus activos hacen frente o son suficientes para cancelar sus pasivos que ha contraído. El resultado correcto sería de 1,5, en donde la solvencia estaría equilibrada para la empresa, si es superior a este valor, los activos de la organización estarían caminado a ser improductivos, para ello se tendrá que tomar la mejor decisión. 
Tabla 3. Ratio de solvencia

\begin{tabular}{ll}
\hline Historial Años & Valor \\
\hline & 1,4 \\
2014 & 1,38 \\
2015 & 1,47 \\
2016 & 1,52 \\
2017 & 1,54 \\
2018 & \\
\hline
\end{tabular}

Fuente: Elaboración propia

Gráfico 4. Análisis del ratio solvencia bajo el enfoque difuso

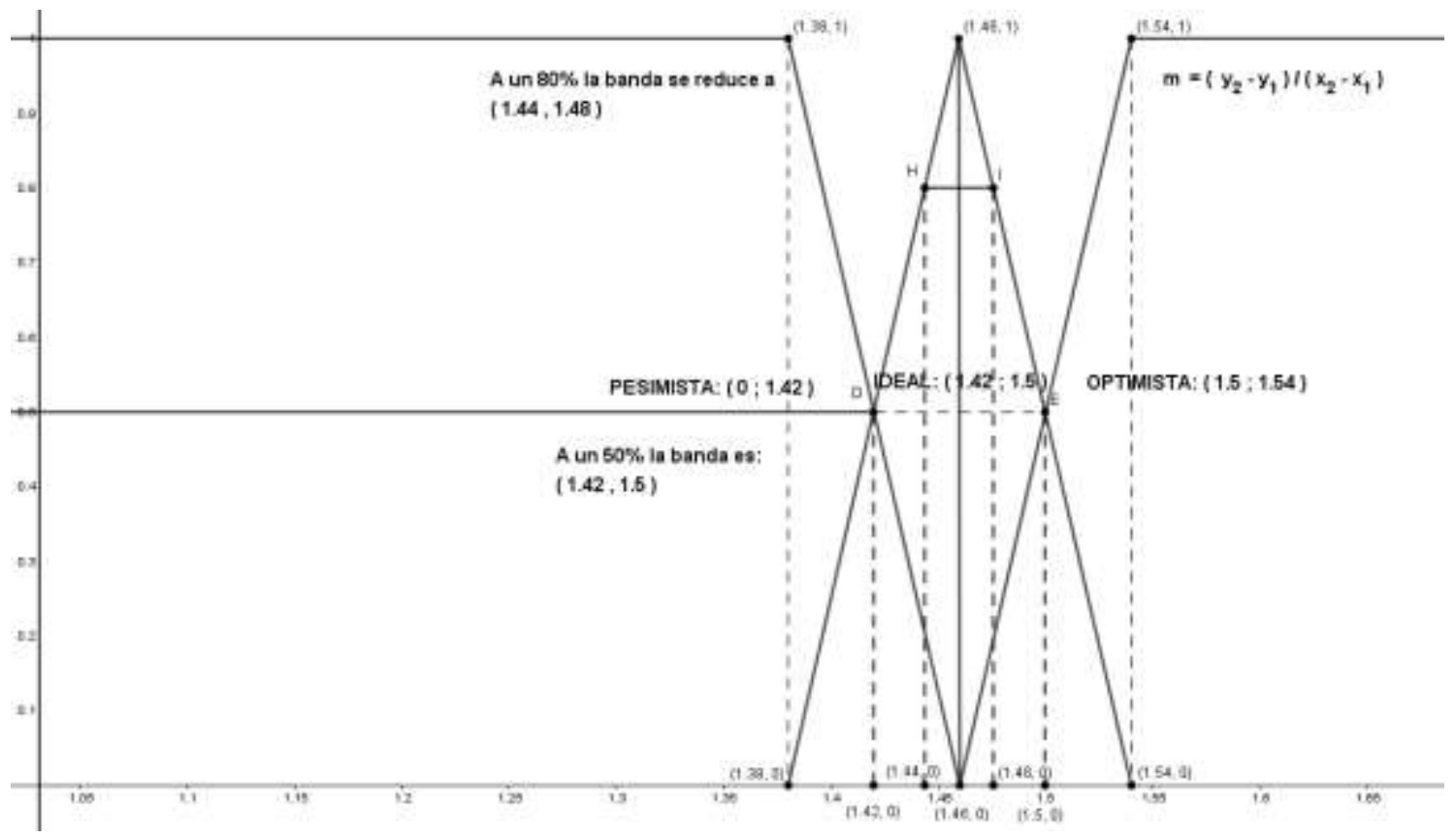

Fuente: Elaboración propia

Con el mismo procedimiento anterior, los valores [1.38, 1,46, 1.54], representan el numero borroso triangular (NBT) del ratio solvencia, el primer extremo pertenece al año 2015, el segundo extremo corresponde al año 2018, y el valor del medio representa el valor de mayor posibilidad de ocurrencia de la razón de solvencia. El análisis de los intervalos de confianza es análogo a los ratios anteriores. 


\section{Resultados}

Los resultados obtenidos, con aplicación de herramientas de avanzada que ofrece la lógica borrosa, demuestran la reducción de la incertidumbre, ambigüedad e imprecisión de los valores. Los intervalos de confianza con alfa cortes del $50 \%[2.35,3.10]$ y otro del $80 \%$ [2.57, 2.87] en la razón corriente, da la posibilidad de entender con mayor precisión la realidad financiera de la empresa. Igual caso ocurre con los indicadores de la prueba acida y solvencia, cuyos intervalos de confianza están entre: $50 \%$ [1.38, 1.62] 80\% [1.45, 1.55]; y, 50\% [1.42, $1.50] 80 \%[1.44,1.48]$ respectivamente.

\section{Conclusiones}

- Realizar un análisis financiero, es interpretar los resultados contables de la empresa con el propósito de obtener un diagnóstico de la realidad actual para poder realizar proyecciones a futuro, con lo cual la gerencia tome las decisiones más acertadas.

- El análisis de los ratios financieros más importantes en sector industrial de la ciudad de Cuenca, con enfoque en la lógica difusa, aplicando herramientas de vanguardia como los números borrosos triangulares (NBT) obtenido de un trazo geométrico triangular y con la utilización de alfa cortes, se logra obtener intervalos de confianza mediante rango de valores, permitiendo reducir la incertidumbre dentro de los valores obtenidos, y estos a su vez se convierten en información más real, rompiendo esquemas tradicionales donde impera la subjetividad, ambigüedad e imprecisión de la información financiera.

- Con este nuevo sistema de control financiero, las empresas industriales de la ciudad de Cuenca-Ecuador, mejorarán su gestión empresarial, tratando de corregir desequilibrios, mitigar riesgos y lograr oportunidades, conllevando a una correcta planificación conociendo la situación real actual de la organización para una eficiente tendencia futura. De esta manera se aporta a la alta gerencia de las empresas de este sector, esta nueva forma de cálculo financiero a través del conocimiento científico, con la finalidad de ampliar sus operaciones con fundamentos de decisión de inversión en resultados de un correcto análisis financiero.

\section{Referencias bibliográficas}

Arimany, N., Farreras, A., y Rabaseda, J. (2016). Análisis económico financiero del sector vinícola de La Rioja en un entorno de crisis. Intangible Capital, 12(1): 268-294. Recuperado de http://www.intangiblecapital.org/index.php/ic/article/view/686/499 
Bernal, D., y Amat, O. (2012). Anuario de ratios financieros sectoriales en México para análisis comparativo empresarial. Ra Ximhai, 8(2), 271-286. Recuperado de http://www.redalyc.org/articulo.oa?id=46125172003

Casanovas, M., y Fernández, A. (2003). La gestión de la tesorería en la incertidumbre. Madrid, España: Ediciones Pirámide.

Díaz, J., Coba, E., Moreno, K., y Santamaría, E. (2017). La lógica difusa aplicada a los ratios financieros en el sector cooperativo del Ecuador. Innova Research Journal, 2(6), 6482. Recuperado de http://revistas.uide.edu.ec/index.php/innova/article/view/215/310

Gutiérrez, J. (2006). Aplicación de los conjuntos borrosos a las decisiones de inversión. Administer 9. Recuperado de https://repository.eafit.edu.co/handle/10784/7689

Kaufmann, A. y Gil, J. (1986). Introducción de la teoría de subconjuntos borrosos a la gestión de las empresas. Madrid, España: Milladoiro.

Kaufmann, A. y Gil, J. (1987). Técnicas operativas de gestión para el tratamiento de la incertidumbre. Barcelona, España: Hispano Europea.

Luna, K., Tinto, J., Sarmiento, W., y Cisneros, D. (2018). Estudio de rentabilidad para el lanzamiento de un nuevo producto aplicando el enfoque difuso. Visión Gerencial, (1), 42-53. Recuperado de: http://www.redalyc.org/articulo.oa?id=465554397005

Medina, S. (2006). Estado de la cuestión acerca del uso de la lógica difusa en problemas financieros, Cuadernos de Administración, XIX (32), 195-223. Recuperado de http://www.scielo.org.co/pdf/cadm/v19n32/v19n32a09.pdf

Nava, A. (2009). Análisis financiero: una herramienta clave para una gestión financiera eficiente. Revista Venezolana de Gerencia, 14(48), 606-628. Recuperado de http://www.redalyc.org/articulo.oa?id=29012059009

Rico, M., y Tinto, J. (2008). Matemática borrosa: algunas aplicaciones en las ciencias económicas, administrativas y contables. Revista de Contaduría, (52), 199-214. Recuperado de http://aprendeenlinea.udea.edu.co/revistas/index.php/cont/article/view/2169

Villegas, E. (2002). Análisis Financiero en los Agronegocios. Revista Mexicana de Agronegocios, 337-346.

Villegas, E., Hernández, M., y Salazar, B. (2017). La medición del capital intelectual y su impacto en el rendimiento financiero en empresas del sector industrial en México. Contaduría y Administración, 62, 184-206. Recuperado de http://www.cya.unam.mx/index.php/cya/article/view/825/854 


\section{PARA CITAR EL ARTÍCULO INDEXADO.}

Luna Altamirano, K., Espinoza González, J., Sarmiento Espinoza, W., Andrade Cordero, C., \& Chamba Esparza, V. (2019). Análisis financiero en el sector industrial con aplicación de herramientas de la lógica borrosa. Ciencia Digital, 3(2.3), 112-124.

https://doi.org/10.33262/cienciadigital.v3i2.3.566

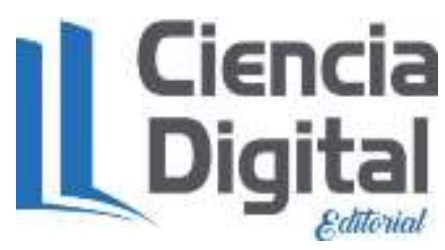

El artículo que se publica es de exclusiva responsabilidad de los autores y no necesariamente reflejan el pensamiento de la Revista Ciencia Digital.

El artículo queda en propiedad de la revista y, por tanto, su publicación parcial y/o total en otro medio tiene que ser autorizado por el director de la Revista Ciencia Digital.
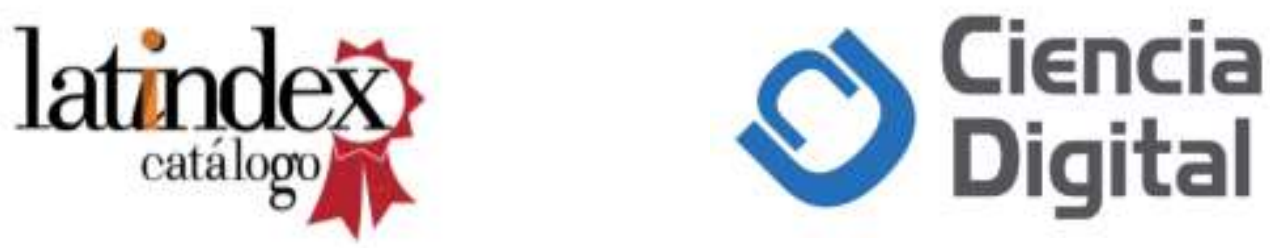